ASC Report No. 41/2011

On the Calculation of the Finite Hankel Transform Eigenfunctions

P. Amodio, T. Levitina, G. Settanni, E.B. Weinmüller 


\section{Most recent ASC Reports}

40/2011 D.P. Hewett, S. Langdon, J.M. Melenk

A high frequency hp boundary element method for scattering by convex polygons

39/2011 A. Jüngel

Semiconductor Device Problems

38/2011 L. Neumann, A. Arnold, W. Hochhauser

Zur Stabilität von geklebten und geklotzten Glasscheiben: Beurteilung der Dunkerley'schen Geraden zur Beulwertbestimmung

37/2011 A. Dick, O. Koch, R. März, E. Weinmüller

Collocation Schemes for Nonlinear Index 1 DAEs with a Singular Point

36/2011 J.A. Carrillo, S. Hittmeir, A. Jüngel

Cross diffusion and nonlinear diffusion preventing blow up in the Keller-Segel model

35/2011 P. Fuchs, A. Jüngel, M. von Renesse

On the Lagrangian structure of quantum fluid models

34/2011 W. Auzinger, O. Koch, M. Thalhammer

Defect-based local error estimators for splitting methods, with application to Schrödinger equations Part I. The linear case

33/2011 A. Jüngel, R. Weishäupl

Blow-up in two-component nonlinear Schrödinger systems with an external driven field

$32 / 2011$ S. Ferraz-Leite, JM. Melenk, D. Praetorius

Numerical quadratic energy minimization bound to convex constraints in thinfilm micromagnetics

31/2011 JM. Melenk, C. Xenophontos, L. Oberbroeckling

Robust exponential convergence of hp-FEM for singularly perturbed reaction diffusion systems with multiple scales

Institute for Analysis and Scientific Computing

Vienna University of Technology

Wiedner Hauptstraße 8-10

1040 Wien, Austria

E-Mail: admin@asc.tuwien.ac.at

WWW: http://www.asc.tuwien.ac.at

FAX: $\quad+43-1-58801-10196$

ISBN 978-3-902627-04-9

(c) Alle Rechte vorbehalten. Nachdruck nur mit Genehmigung des Autors.

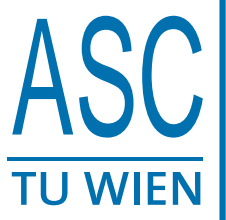




\title{
On the Calculation of the Finite Hankel Transform Eigenfunctions
}

\author{
P. Amodio, T. Levitina, G. Settanni, and E. B. Weinmüller
}

November 29, 2011

\begin{abstract}
In the recent years considerable attention has been focused on the numerical computation of the eigenvalues and eigenfunctions of the finite (truncated) Hankel transform, important for numerous applications. However, due to the very special behavior of the Hankel transform eigenfunctions, their direct numerical calculation often causes an essential loss of accuracy.

Here, we discuss several simple, efficient and robust numerical techniques to compute Hankel transform eigenfunctions via the associated singular self-adjoint Sturm-Liouville operator. The properties of the proposed approaches are compared and illustrated by means of numerical experiments.
\end{abstract}

Key words: Finite Hankel Transform, generalized spheroidal wave functions, singular Sturm-Liouville problem, finite difference schemes, Prüfer angle.

MSC 2000: 34B16, 34B24, 34L16, 65F15, 65L10, 65L15, 65R10, 65R20

\section{Introduction}

During the last fifty years a lot of effort has been put into the investigation of the eigenvalues and eigenfunctions of the finite (truncated) Fourier transform (FFT). This strong interest in the structure and in the numerical simulation of the related eigenvalue problems is motivated by important applications. The eigenfunctions are first defined on a bounded region, say a $n$-dimensional ball $B_{a}^{n}$ of radius $a$, to which the Fourier transform integration is truncated. Then, each eigenfunction can be extended in accordance with its Fourier transform to the whole space $\mathbb{R}^{n}$. Consequently, we deal with a function whose Fourier transform has a finite support confined to the ball $B_{a}^{n}$. 
The increasing interest in those functions can be explained by their properties. It was shown in $[29,30]$ that although extended to the whole space $\mathbb{R}^{n}$, the FFT eigenfunctions remain mostly localized inside $B_{a}^{n}$. For instance, the eigenfunction corresponding to the largest eigenvalue of the FFT provides the maximum of the ratio $\|\Psi\|_{B_{a}^{n}} /\|\Psi\|_{\mathbb{R}^{n}}$ over the space of functions whose Fourier transforms are confined to $B_{a}^{n}$. Here, by $\|\cdot\|_{B_{a}^{n}}$ and $\|\cdot\|_{\mathbb{R}^{n}}$ the $\mathcal{L}_{2}$ norms in $\mathcal{L}_{2}\left(B_{a}^{n}\right)$ and $\mathcal{L}_{2}\left(\mathbb{R}^{n}\right)$ are denoted, respectively. Therefore, the convolution of the above eigenfunction with a $n$-dimensional signal can be truncated to $B_{a}^{n}$ without an essential loss of the signal information. In a 2D-case, the eigenfunctions of the Fourier transform truncated to a circle of radius $a$ can be expressed using the eigenfunctions of the finite (truncated) Hankel transform (FHT), i.e. non-trivial solutions of the integral equation

$$
\mu T(\eta)=\int_{0}^{1} \sqrt{a^{2} \tilde{\eta} \eta} J_{m}\left(a^{2} \tilde{\eta} \eta\right) T(\tilde{\eta}) \mathrm{d} \tilde{\eta}, \quad \eta \in(0,1),
$$

where $\mu$ is the associated eigenvalue and $J_{m}(\xi)$ is the Bessel function of the 1st kind and index $m, m=0,1,2, \ldots$

FHT eigenfunctions were first introduced in [16, 32] and applied for the calculation of mode patterns and losses in optical resonators with circular mirrors of spherical curvatures. Practically, at the same time, they were used in [29] in context of the signal/image processing, where their properties were studied. Moreover, a numerical technique for their evaluation via infinite series containing Jacobi polynomials and Bessel functions were presented. Later, even more detailed analysis of the FHT eigenfunction properties was given in [22]. Since that time they became a powerful tool in the numerical optical analysis, as well as in the spectral estimation of the 2D-processes. It is hardly possible to survey all their applications in a short paper. FHT eigenfunctions are employed for an optimal antenna synthesis and for resolution enhancing of an optical system (see e.g. [3, 4, 11, 12, 27]). FHT eigenfunctions constitute a complete basis in $\mathcal{L}_{2}(0,1)$; extended to $\mathbb{R}_{+}$ they form a basis in the class of Hankel band-limited functions. Therefore, they serve in medicine and biology for tomographic image reconstruction $[20,26,31]$ and in astrophysics for both forward and inverse analysis of high numerical aperture focusing systems $[9,28]$.

Although these functions are so important for practical purposes in various fields, they are not often used in practice. The reason is that they cannot be expressed in a closed form using polynomials and/or standard hypergeometric functions, and thus, they can only be evaluated numerically. 
Most of the known numerical techniques for FHT eigenfunction evaluation utilize their asymptotic behavior valid either for $a \rightarrow 0$ or for $a \rightarrow \infty$ [10]. Often various discretizations of the integral in the right hand side of (1) are provided, see examples in $[18,19,24,33]$. Yet, the direct computation based on (1) is hardly possible for a wide range of parameters, since the associated eigenvalues $\mu$, though proved to be simple, may be located very close to each other, being indistinguishable numerically, see Table 1 in [18]. Even, when we succeeded to calculate few first of them, the remaining ones are practically equal zero and notoriously difficult to compute with a reasonable accuracy.

The direct approach, using (1), not simply spoils the accuracy, but in this case the 'numerical eigenvalues' are no more simple and, as a consequence, the 'numerical eigenfunctions' are unpredictable linear combinations of the actual eigenfunctions - just any vector from the subspace spanned by the eigenvectors corresponding to almost coinciding eigenvalues. This may cause especially much damage while sampling with the FHT eigenfunctions, where the Fourier coefficients of the recovered function $f$ are expressed not via the scalar products $\left\langle f, T_{m l}\right\rangle$, but rather via the samples of $f$.

Alternatively, and we shall pursue this approach in this paper, the FHT eigenfunctions can be computed as eigenfunctions of a self-adjoint Sturm-Liouville problem (3), since the involved differential operator defining this problem commutes with the FHT operator. For details we refer to [21, 22, 29]. However, since the Sturm-Liouville problem is singular, its numerical treatment requires additional attention: Especially, the boundary conditions have to be formulated in a proper form to guarantee that the resulting eigenvalue problem is well-posed. The discussion of the correctly posed boundary conditions will be provided in the next section of the paper.

In the literature, several ways to solve singular eigenvalue problems numerically are proposed. In [17] the following class of eigenvalue problems has been considered:

$$
y^{\prime}(t)-\frac{M}{t} y(t)-A(t) y(t)=\lambda G(t) y(t), \quad t \in(0,1], \quad y \in C[0,1] \cap C^{1}(0,1],
$$

where $M \in \mathbb{R}^{n \times n}$ and $A(t), G(t) \in \mathbb{R}^{n \times n}$ are continuous matrix functions of $t \in[0,1]$. Especially, the structure of the boundary conditions $B_{0} y(0)+B_{1} y(1)=0$ necessary and sufficient for the eigenfunction $y$ to satisfy $y \in C[0,1] \cap C^{1}(0,1]$ has been studied. Moreover, the solution structure of the eigenvalue problems has been described, the box-scheme has been applied for its numerical solution, and a convergence result for this 
method has been given. Similar class of problems have been discussed in [8]. Here, the eigenvalue problems for ordinary differential equations are recast in a formulation suitable for the solution by polynomial collocation. It is shown that the well-posedness of the two formulations is equivalent in the regular as well as in the singular case. Thus, a collocation code equipped with asymptotically correct error estimation and adaptive mesh selection can be successfully applied to compute the eigenvalues and eigenfunctions efficiently and with reliable control of the accuracy. This approach has been used in [15] to solve eigenvalue problems for singular Schrödinger equations arising, for example, in electronic structure computations. Here, first rough approximations by a matrix method are generated; these approximations are used as starting values for an adaptive collocation method which yields approximations of high accuracy together with a reliable error estimate. This method has been successfully applied to the solution of the coupled ODE Stark problem for the hydrogen atom.

Another approach to Sturm-Liouville problems has been presented in [13], where high order finite difference schemes are used to approximate the involved derivatives of the continuous problem $(3),[5,6,7]$. Here, the second order problem is treated directly and it is not transformed into a first order form, which helps to safe computational effort. See also discussion in [23] for an alternative solution method.

Concerning the numerical simulations presented here, they will be first of all compared with calculations from [29]. Moreover, their correctness will be also justified using equation (1) which indeed turns out to be a very reliable benchmark. Note that (1) also enables to extend an eigenfunction analytically to the whole semi-axis $\mathbb{R}_{+}$.

\section{Generalized prolate spheroidal functions}

Unlike the 1D-case the eigenvalue problem for the multidimensional FFT may be formulated in many ways depending on the finite domain to which the entire 2D-plane is truncated. However, the circular domain $r=\|\vec{r}\|_{2} \leq a$ seems to be most important for applications in astrophysics, tomography, etc. In this case the FFT eigenfunctions $\psi(\vec{r})$ and the associated eigenvalues $\chi$ are defined by the integral equation

$$
\chi \psi(\vec{r})=\int_{r=\|\vec{r}\|_{2} \leq a} \mathrm{e}^{i(\vec{r}, \vec{v})} \psi(\vec{v}) \mathrm{d} \vec{v} .
$$

Following considerations in [29], we change to polar coordinates $(\varphi, \eta)$ and, 
due to the symmetry of a circle, we can reduce the above problem to a onedimensional formulation for the radial component of an eigenfunction $\psi_{m l}(\vec{r})$, $m=0,1,2, \ldots, l=0,1,2, \ldots$,

$$
\mu_{m l} T_{m l}(\eta)=\int_{0}^{1} \sqrt{c \eta \rho} J_{m}(c \eta \rho) T_{m l}(\rho) \mathrm{d} \rho,
$$

where the related angular component is then $\mathrm{e}^{ \pm i m \varphi}$. In the above formulation, $c:=a^{2}$ and the eigenvalues $\mu_{m l}$ are ordered by magnitude, $\left|\mu_{m 0}\right|>\left|\mu_{m 1}\right| \ldots>0$. The integral operator

$$
H[f](\eta)=\int_{0}^{1} \sqrt{c \eta \rho} J_{m}(c \eta \rho) H[f](\rho) \mathrm{d} \rho
$$

can be interpreted as a FHT, and therefore, we call $T_{m l}$ the FHT eigenfunctions.

These functions are also often referred to as hypospheroidal, or generalized prolate spheroidal functions (GPSF), due to their similarity in behaviour and main properties with the prolate spheroidal wave functions, see e.g. [21] and the references therein. Thus, as was shown in [29], cf. [21, 22], $T_{m l}$ are eigenfunctions of a singular self-adjoint Sturm-Liouville problem. This means that they are non-trivial solutions of the equation

$$
\frac{\mathrm{d}}{\mathrm{d} \eta}\left(\left(1-\eta^{2}\right) \frac{\mathrm{d}}{\mathrm{d} \eta} T(\eta)\right)+Q(\eta) T(\eta)=0, Q(\eta)=\lambda-c^{2} \eta^{2}-\frac{m^{2}-1 / 4}{\eta^{2}}
$$

defined on the interval $\eta \in I=(0,1)$ and bounded at its ends,

$$
|T(\eta)|<\infty, \eta \rightarrow 0+, \quad|T(\eta)|<\infty, \eta \rightarrow 1-.
$$

As usual, values $\lambda=\lambda_{m l}$ admitting non-trivial solutions $T_{m l}(\eta)$ of (3), (4) are the associated eigenvalues; one can prove that $\lambda_{m 0}<\lambda_{m 1}<\ldots$ and $l$ is the number of zeros the eigenfunction $T_{m l}(\eta)$ has inside $I$.

For the numerical treatment, we shall normalize the eigenfunctions by the requirement

$$
\int_{0}^{1}\left|T_{m l}(\eta)\right|^{2} \mathrm{~d} \eta=1 .
$$

Before proceeding, we have to reformulate the boundedness conditions (4) in a form suitable for the numerical treatment. At both ends of the interval $I$ the problem exhibits singularities of the 1st kind. In order to describe the 
asymptotical behavior of the bounded solutions we have to look at the general solution of the problem,

$$
\frac{\mathrm{d}}{\mathrm{d} \eta}\left(\left(1-\eta^{2}\right) \frac{\mathrm{d}}{\mathrm{d} \eta} T(\eta)\right)-\left(c^{2} \eta^{2}+\frac{m^{2}-1 / 4}{\eta^{2}}\right) T(\eta)=0,
$$

or equivalently, at the roots of the so called characteristic polynomial. At $\eta=0$ these roots are $m \pm 1 / 2$, while at $\eta=1$ they both vanish. According to $[14,22]$, any solution of (3) which is bounded for $\eta \rightarrow 0+$ has the form

$$
T(\eta)=\eta^{m+1 / 2} W(\eta),
$$

where $W(\eta)$ is an analytic function and $W(0) \neq 0$ holds. This means that the necessary and sufficient condition any bounded solution has to satisfy, reads, cf. [17],

$$
T(0)=0 .
$$

Note that the representation (6) also describes the smoothness of the solution $T(\eta)$ at $\eta=0$ and clearly, the smoothness of $T(\eta)$ depends on $m$. Naturally, and this can be observed in the computation, lack of smoothness in the higher solution derivatives usually causes order reductions in the numerical methods and consequently, the loss of their efficiency. In order to avoid this loss, one can alternatively transfer the boundary condition (7) to a nearby point, where the solution $T(\eta)$ remains an analytic function.

Due to the representation (6), we expect that unlike $T(\eta)$, the ratio $\eta T^{\prime}(\eta) / T(\eta)$ is analytic at $\eta=0$ and can therefore be expressed in the form of a power series,

$$
\eta\left(1-\eta^{2}\right) \frac{T^{\prime}(\eta)}{T(\eta)}=\mathcal{A}(\eta)=\sum_{k=0}^{\infty} \alpha_{k} \eta^{2 k} .
$$


According to (3), the coefficients $\alpha_{k}$ are

$$
\begin{aligned}
& \alpha_{0}=m+\frac{1}{2}, \\
& \alpha_{1}=\frac{-\alpha_{0}^{2}-\lambda}{1+2 \alpha_{0}} \\
& \alpha_{2}=\frac{\alpha_{1}-\alpha_{1}^{2}+c^{2}+\lambda}{3+2 \alpha_{0}}, \\
& \alpha_{3}=\frac{3 \alpha_{2}-2 \alpha_{2} \alpha_{1}-c^{2}}{5+2 \alpha_{0}}, \\
& \alpha_{k}=\frac{(2 k-3) \alpha_{k-1}-\sum_{j=1}^{k-1} \alpha_{j} \alpha_{k-j}}{2 k-1+2 \alpha_{0}}, \quad k=4,5, \ldots .
\end{aligned}
$$

Let $A:=\max \left\{1,\left|\alpha_{1}\right|^{1 / 2},\left|\alpha_{2}\right|^{1 / 4},\left|\alpha_{3}\right|^{1 / 6}\right\}$. Then using complete induction, one can easily show that

$$
\left|\alpha_{k}\right| \leq \frac{3}{2} A^{2 k}, \quad k=4,5, \ldots
$$

To see this, it is sufficient to note that

$$
\left|\alpha_{4}\right|=\left|\frac{5 \alpha_{3}-2 \alpha_{1} \alpha_{3}-\alpha_{4}^{2}}{7+2 \alpha_{0}}\right| \leq \frac{1}{7}\left(5 A^{6}+3 A^{8}\right) \leq \frac{3}{2} A^{8},
$$

and then, under assumption that estimate (10) holds for all $k<n$, to show that it is also true for $k=n$. Indeed

$$
\left|\alpha_{n}\right|=\left|\frac{(2 n-3) \alpha_{n-1}-\sum_{j=1}^{n-1} \alpha_{j} \alpha_{n-j}}{2 n-1+2 \alpha_{0}}\right| \leq \frac{\left(2 n A^{2(n-1)}+n A^{2 n}\right)}{2 n} \leq \frac{3}{2} A^{2 n} .
$$

Thus, the series in (8) converges uniformly and absolutely on an interval $\left[0, \delta_{0}\right]$, where $A \delta_{0}<1$ holds, and this justifies the above manipulations. Furthermore, since for $\eta \in\left(0, \delta_{0}\right]$

$$
\left|\sum_{k=N+1}^{\infty} \alpha_{k} \eta^{2 k}\right| \leq \frac{3}{2} \sum_{k=N+1}^{\infty}\left(A \delta_{0}\right)^{2 k} \leq\left(A \delta_{0}\right)^{N+1} \frac{3}{2\left(1-A \delta_{0}\right)}
$$


the series (8) truncated to $N_{0}$ terms,

$$
N_{0} \geq \frac{\ln \left(\frac{2}{3} \varepsilon\left(1-A^{2} \delta_{0}^{2}\right)\right)}{2 \ln \left(A \delta_{0}\right)},
$$

approximates the exact value of $\mathcal{A}(\eta)$ with the accuracy of $\varepsilon$,

$$
\left|\mathcal{A}(\eta)-\sum_{k=0}^{N_{0}} \alpha_{k} \eta^{2 k}\right|<\varepsilon,
$$

uniformly on $0 \leq \eta \leq \delta_{0}$.

At the point $\eta=1$ the bounded solution is analytic, however it does not vanish there and any Dirichlet boundary condition imposed on the solutions of (3) at $\eta=1$ is not the boundedness condition (4). Instead, again the logarithmic derivative will be used. We expand the function

$$
\mathcal{B}(\eta)=\left(1-\eta^{2}\right) \frac{d}{d \eta} T(\eta) / T(\eta),
$$

in the power series

$$
\mathcal{B}(\eta)=\sum_{k=1}^{\infty} \beta_{k}(1-\eta)^{k}
$$

with the coefficients

$$
\begin{aligned}
& \beta_{1}=\lambda-c^{2}-\left(m^{2}-1 / 4\right) \\
& \beta_{2}=\frac{1}{4}\left(\beta_{1}+\beta_{1}^{2}-3\left(m^{2}-1 / 4\right)+5 c^{2}-\lambda\right), \\
& \beta_{3}=\frac{1}{6}\left(2 \beta_{2}+2 \beta_{1} \beta_{2}-4\left(m^{2}-1 / 4\right)-4 c^{2}\right), \\
& \beta_{4}=\frac{1}{8}\left(3 \beta_{3}+2 \beta_{1} \beta_{3}+\beta_{2}^{2}-5\left(m^{2}-1 / 4\right)+c^{2}\right), \\
& \beta_{k}=\frac{1}{2 k}\left((k-1) \beta_{k-1}+\sum_{j=1}^{k-1} \beta_{j} \beta_{k-j}-(k+1)\left(m^{2}-1 / 4\right)\right), \quad k=5,6, \ldots
\end{aligned}
$$

As before, the series given in (14) converges uniformly and absolutely on the interval $\left[1-\delta_{1}, 1\right]$, provided that

$$
B \delta_{1}<1, \quad B:=\max \left\{2\left(m^{2}-\frac{1}{4}\right),\left|\beta_{1}\right|,\left|\beta_{2}\right|^{1 / 2},\left|\beta_{3}\right|^{1 / 3},\left|\beta_{4}\right|^{1 / 4}\right\} .
$$


Moreover, for a given $\varepsilon>0$, the number of terms $N_{1}$ can be determined in such a way that

$$
\left|\mathcal{B}(\eta)-\sum_{k=1}^{N_{1}} \beta_{k}(1-\eta)^{k}\right|<\varepsilon, \quad \forall \eta \in\left[1-\delta_{1}, 1\right],
$$

holds. Here,

$$
N_{1} \geq \frac{\ln \left(\frac{2}{3} \varepsilon\left(1-B \delta_{1}\right)\right)}{\ln \left(B \delta_{1}\right)} .
$$

Now, it is clear how to pose the boundary condition at $\eta=1$,

$$
2 T^{\prime}(1)=\beta_{1} T(1)=Q(1) T(1) .
$$

Similar to the case $\eta=0$, relation (13) can be used to transfer the boundary condition from $\eta=1$ to a nearby regular point. Hereafter $m$ and $l$ are fixed integers and we are computing the eigenfunction $T_{m l}(\eta)$ of the multi-index $(m, l)$ and its associated eigenvalue $\lambda_{m l}$.

\section{$3 \quad$ Numerical schemes}

We now discuss how we discretize the differential equation (3) subject to

(5) and suitably chosen boundary conditions. Here, we restrict ourselves to equidistant grids of the form

$$
\Delta_{h}:=\left\{0 \leq \eta_{0}<\eta_{1}<\cdots<\eta_{n} \leq 1, \eta_{i}=\eta_{0}+i h, i=1, \ldots, n\right\}
$$

where $h:=\left(\eta_{n}-\eta_{0}\right) / n$. The solution derivatives in the inner grid points are approximated by central difference formulae of high order, cf. [5],

$$
T_{m l}^{(\nu)}\left(\eta_{i}\right) \approx T_{i}^{(\nu)}=\frac{1}{h^{\nu}} \sum_{j=-s}^{s} \alpha_{j+s}^{(\nu)} T_{i+j}, \quad \nu=1,2,
$$

where $T_{i} \approx T_{m l}\left(\eta_{i}\right)$. The coefficients $\alpha_{j+s}^{(\nu)}$ are chosen by a series expansion of $T_{i+j}$ in $\eta_{i}, i=-s, \ldots, s$. In such a way the resulting formula has order of consistency $k=2 s$ provided that the solution is sufficiently smooth. For example, for a formula of order $k=4$, we have

$$
\begin{aligned}
& \left(\alpha_{0}^{\prime}, \ldots, \alpha_{4}^{\prime}\right)=\left(\frac{1}{12},-\frac{2}{3}, 0, \frac{2}{3},-\frac{1}{12}\right), \\
& \left(\alpha_{0}^{\prime \prime}, \ldots, \alpha_{4}^{\prime \prime}\right)=\left(-\frac{1}{12}, \frac{4}{3},-\frac{5}{2}, \frac{4}{3},-\frac{1}{12}\right) .
\end{aligned}
$$


Symmetric formulae (19) can be used in (18) for the grid points $i=s, \ldots, n-s$. For the approximations of $T_{m l}^{(\nu)}\left(\eta_{i}\right)$ in the grid points $i=0, \ldots, s-1$ and $n-s+1, \ldots, n$, we cannot use the symmetric stencils. Instead, nonsymmetric finite differences are considered. For example, the following approximations

$$
\begin{aligned}
& T_{0}^{\prime}=\frac{1}{h}\left(-\frac{25}{12} T_{0}+4 T_{1}-3 T_{2}+\frac{4}{3} T_{3}-\frac{1}{4} T_{4}\right) \\
& T_{1}^{\prime}=\frac{1}{h}\left(-\frac{1}{4} T_{0}-\frac{5}{6} T_{1}+\frac{3}{2} T_{2}-\frac{1}{2} T_{3}+\frac{1}{12} T_{4}\right) \\
& T_{0}^{\prime \prime}=\frac{1}{h^{2}}\left(\frac{15}{4} T_{0}-\frac{77}{6} T_{1}+\frac{107}{6} T_{2}-13 T_{3}+\frac{61}{12} T_{4}-\frac{5}{6} T_{5}\right), \\
& T_{1}^{\prime \prime}=\frac{1}{h^{2}}\left(\frac{5}{6} T_{0}-\frac{5}{4} T_{1}-\frac{1}{3} T_{2}+\frac{7}{6} T_{3}-\frac{1}{2} T_{4}+\frac{1}{12} T_{5}\right),
\end{aligned}
$$

have order of consistency $k=4$. The coefficients of the finite differences used for the approximation of the respective derivatives in the vicinity of the interval end may be symmetric or antisymmetric to those given above.

For a general second order ODE we approximate each derivative separately by the previous formulae, so that a discrete problem in the unknowns $T_{i}$, $i=0,1, \ldots, n$, results. As shown below, for BVPs we will consider two possible choices of boundary conditions to close the discrete problem.

For regular second order BVPs for ODEs with appropriately smooth solution, the global error of the proposed difference scheme is $O\left(h^{k}\right)$, where $k$ is the order of consistency, [7]. For Sturm-Liouville problems the obtained discrete system (finite difference scheme and boundary conditions) constitutes an algebraic eigenvalue problem and can be solved using the MatLaB function eigs which provides the absolute values of a certain number of eigenvalues and the corresponding eigenvectors. These eigenvectors are the values of the eigenfunctions in the grid points. The eigenfunctions are finally scaled using condition in (5), where the integral is approximated by the trapezoidal rule.

Another option to approach the problem is to augment the discretization of (3) by boundary conditions and the discrete version of (5) provided via the trapezoidal rule. Such a nonlinear eigenvalue problem can be solved for an eigenvalue and the associated normalized eigenfunction by means of the Newton method. 


\subsection{Approach 1}

Here, we solve (3) and (5) subject to boundary conditions

$$
T_{m l}(0)=0, \quad 2 T_{m l}^{\prime}(1)=Q(1) T_{m l}(1) .
$$

We discretize the boundary conditions (21) and the differential equation (3) in the grid points $\eta_{i}, i=1, \ldots, n-1$, and obtain the system

$$
\left(\begin{array}{cc}
1 & 0 \\
m_{0} & M
\end{array}\right)\left(\begin{array}{c}
T_{0} \\
\mathbf{T}
\end{array}\right)=-\lambda_{m, l}\left(\begin{array}{c}
0 \\
\mathbf{T}
\end{array}\right),
$$

where $m_{0}$ is a column vector of length $n$ containing weights associated with $T_{0}, M$ is a square $n \times n$ matrix, and $\mathbf{T}=\left(T_{1}, \ldots, T_{n}\right)^{T}$. Note that the second boundary condition is the limiting version of the differential equation for $\eta=1$ and therefore the discrete version of this boundary condition is identical with the discretization of (3) at $\eta_{n}$.

As a consequence of the foregoing section, the rectangular $n \times(n+1)$ matrix $\left(m_{0} M\right)$ has the form

$$
\left(m_{0} M\right)=D_{1} A+D_{2} B+D_{3}(0 I),
$$

where $D_{1}, D_{2}$ and $D_{3}$ are diagonal $n \times n$ matrices with entries

$$
d_{i 1}=1-\eta_{i}^{2}, \quad d_{i 2}=-2 \eta_{i}, \quad d_{i 3}=c^{2}\left(1-\eta_{i}^{2}\right)-\frac{m^{2}-1 / 4}{\eta_{i}^{2}}, \quad i=1, \ldots, n,
$$

respectively, 0 is a column zero vector of dimension $n$, and $I$ is a $n \times n$ identity matrix. Moreover, $A$ and $B$ are the $n \times(n+1)$ matrices containing the coefficients of the methods. For the order $k=4$ they have the form,

$$
A=\frac{1}{h^{2}}\left(\begin{array}{rrrrrrrrr}
\frac{5}{6} & -\frac{5}{4} & -\frac{1}{3} & \frac{7}{6} & -\frac{1}{2} & \frac{1}{12} & & & \\
-\frac{1}{12} & \frac{4}{3} & -\frac{5}{2} & \frac{4}{3} & -\frac{1}{12} & & & & \\
& -\frac{1}{12} & \frac{4}{3} & -\frac{5}{2} & \frac{4}{3} & -\frac{1}{12} & & & \\
& & \ddots & \ddots & \ddots & \ddots & \ddots & & \\
& & & \ddots & \ddots & \ddots & \ddots & \ddots & \\
& & & & -\frac{1}{12} & \frac{4}{3} & -\frac{5}{2} & \frac{4}{3} & -\frac{1}{12} \\
& & & \frac{1}{12} & -\frac{1}{2} & \frac{7}{6} & -\frac{1}{3} & -\frac{5}{4} & \frac{5}{6} \\
& & & -\frac{5}{6} & \frac{61}{12} & -13 & \frac{107}{6} & -\frac{77}{6} & \frac{15}{4}
\end{array}\right)
$$




$$
B=\frac{1}{h}\left(\begin{array}{rrrrrrrrr}
-\frac{1}{4} & -\frac{5}{6} & \frac{3}{2} & -\frac{1}{2} & \frac{1}{12} & & & & \\
\frac{1}{12} & -\frac{2}{3} & 0 & \frac{2}{3} & -\frac{1}{12} & & & & \\
& \frac{1}{12} & -\frac{2}{3} & 0 & \frac{2}{3} & -\frac{1}{12} & & & \\
& & \ddots & \ddots & \ddots & \ddots & \ddots & & \\
& & & \ddots & \ddots & \ddots & \ddots & \ddots & \\
& & & & \frac{1}{12} & -\frac{2}{3} & 0 & \frac{2}{3} & -\frac{1}{12} \\
& & & & -\frac{1}{12} & \frac{1}{2} & -\frac{3}{2} & \frac{5}{6} & \frac{1}{4} \\
& & & & \frac{1}{4} & -\frac{4}{3} & 3 & -4 & \frac{25}{12}
\end{array}\right)
$$

the approximation of (3) in point $\eta_{1}$, which means that the entries of the first rows of the matrices $A$ and $B$ are obtained by a series expansion of $T_{i}$ in $\eta_{1}$, $i=0,1, \ldots, k+1$.

Since $T_{0}=0$ the above problem reduces to an eigenvalue problem for the matrix $M$,

$$
M \mathbf{T}=-\lambda_{m l} \mathbf{T},
$$

which is now solved using the algebraic method. Finally, the discrete eigenfunction is normalized using the discrete version of (5) based on the trapezoidal rule.

\subsection{Approach 2}

Now, we set $\eta_{0}=\eta_{a}>0, \eta_{n}=1$ and consider the differential equation (3) augmented by the boundary conditions

$$
\eta_{0}\left(1-\eta_{0}^{2}\right) T^{\prime}\left(\eta_{0}\right)=A\left(\eta_{0}\right) T\left(\eta_{0}\right), \quad 2 T^{\prime}(1)=Q(1) T(1) .
$$

Note, that $A\left(\eta_{0}\right)$ depends nonlinearly on $\lambda$. The finite difference scheme for (3) and (27) yields a discrete system for $T_{0}, T_{1}, \ldots, T_{n}$ involving $\lambda$,

$$
M\left(\begin{array}{c}
T_{0} \\
\mathbf{T}
\end{array}\right)=-\lambda \mathbf{T}
$$

where $M=D_{1} A+D_{2} B+D_{3}(0 I)$ is a $n \times(n+1)$ matrix, with $D_{1}, D_{2}, D_{3}$, $A$ and $B$ specified in (23), (24) and (25), respectively.

Finally, we complete the system by the discrete version of the normalization condition (5) and obtain a nonlinear system of $n+2$ equations for the unknowns 
$T_{0}, \ldots, T_{n}$ and $\lambda$ which is solved by means of Newton iteration. Starting values for the Newton iteration are provided by the approximation obtained from the solution of the related algebraic eigenvalue problem.

\subsection{Approach 3}

An alternative approach, which was originally proposed in [23], is based on the same ideas that lay in the foundation of the technique presented in $[1,2]$ for evaluation of wave functions of prolate spheroids and triaxial ellipsoids.

\subsubsection{Modified Prüfer angle}

The key role in the computations is played by the modified Prüfer angle $\Theta(\eta)$ associated with a solution $T(\eta)$ of $(3)$,

$$
\left(1-\eta^{2}\right) \frac{\mathrm{d}}{\mathrm{d} \eta} T(\eta) / T(\eta)=-\nu(\eta)^{2} \tan (\Theta) .
$$

Here, the scaling function $\nu(\eta)$ serves to make the Prüfer angle $\Theta(\eta)$ smoother and slower changing. The latter two factors are important for the numerical integration of the ODE defining the Prüfer angle,

$$
\begin{aligned}
\frac{\mathrm{d}}{\mathrm{d} \eta} \Theta & =-\frac{2 \nu^{\prime}(\eta) \sin (\Theta) \cos (\Theta)}{\nu(\eta)} \\
& +\frac{\nu^{2}(\eta) \sin ^{2}(\Theta)}{\left(1-\eta^{2}\right)}+\frac{\mathrm{Q}(\eta) \cos ^{2}(\Theta)}{\nu^{2}} .
\end{aligned}
$$

We suggest the following choice of $\nu(\eta)$, for motivation see $[2,25]$,

$$
\nu(\eta)=\left\{\begin{array}{cc}
{\left[\left(1-\eta^{2}\right) Q(\eta)\right]^{1 / 4},} & |Q(\eta)|>1 \\
1, & \text { otherwise }
\end{array}\right.
$$

A remarkable property of a Prüfer angle is that it can be used to count zeros of the corresponding solution of (3). For proof it is sufficient to note that according to (28), at each node $\eta$, where $T(\eta)=0$, the Prüfer angle intersects a horizontal line $\Theta=\frac{2 n+1}{2} \pi, n \in \mathbb{Z}$, increasing at this point, since the right-hand side of (29) is strictly positive.

Moreover, it follows from the analysis of singularities of (3) that at any of the ends of the interval $I=(0,1)$ the subspace of bounded solutions is 
one-dimensional. As a result, all solutions bounded at $\eta=0(\eta=1)$ possess the same logarithmic derivative $\mathrm{d} T(\eta) / \mathrm{d} \eta$ and hence the same Prüfer angle.

Furthermore, since for a fixed $\lambda$ all Prüfer angles satisfy the same first order differential equation, the trajectories of any two of them either do not intersect, or coincide at each point of the interval $I$. In particular the latter holds for the Prüfer angles $\Theta^{i}(\eta), i=0,1$, associated with solutions of (3) bounded at $\eta=0$ and $\eta=1$, respectively.

Comparing relations (8) and (28), one concludes that the Prüfer angle $\Theta^{0}(\eta)$ is at a near point $\eta \in\left(0, \delta_{0}\right], A \delta_{0}<1$,

$$
\Theta^{0}(\eta)=-\arctan \left[\frac{\mathcal{A}(\eta)}{\eta \nu^{2}(\eta)}\right]+n_{0} \pi,
$$

and this relation at the point $\eta=\delta_{0}$ will be used as an initial condition for $\Theta^{0}(\eta)$. Evidently, the Prüfer angle is defined up to a constant $n_{0} \pi, n_{0}=$ $0, \pm 1, \pm 2, \ldots$. In the further we set $n_{0}=0$.

Due to (13), the initial condition for $\Theta^{1}(\eta)$ can be written at a point $\eta=1-\delta_{1}$, $B \delta_{1}<1$, in the following form:

$$
\Theta^{1}(\eta)=-\arctan \left[\frac{\mathcal{B}(\eta)}{\nu^{2}(\eta)}\right]+n_{1} \pi, \quad n_{1}=0, \pm 1, \pm 2, \ldots
$$

We shall require $n_{1}=l \pi$. From the properties mentioned above, it follows that $\lambda=\lambda_{m l}$ if and only if the trajectories $\Theta^{0}(\eta)$ and $\Theta^{1}(\eta)$ defined by the initial conditions (30) and (31), respectively, coincide on $I$.

Moreover, if $\lambda \neq \lambda_{m l}$, the sign of the difference $\Delta(\eta)=\Theta^{0}(\eta)-\Theta^{1}(\eta)$ does not change within the interval $I$ and indicates the interposition of $\lambda$ and $\lambda_{m l}$,

$$
\operatorname{sign} \Delta(\eta)=\operatorname{sign}\left(\lambda-\lambda_{m l}\right), \quad \eta \in I .
$$

The proof literally repeats the arguments given in [25] in context of the evaluation of prolate spheroidal functions. The latter equality is established in the case of $\nu(\eta) \equiv 1$ and then the general case follows from the fact that the intersection points of the Prüfer angle with the horizontal lines $\Theta=\frac{2 n+1}{2} \pi$ are independent of the choice of $\nu(\eta)$.

Thus the algorithm detecting the eigenvalue $\lambda_{m l}$ of a desired multi-index $(m, l)$ is straightforward. Based on the sign of the difference between $\Theta^{0}(\eta)=\Theta_{\lambda}^{0}(\eta)$ and $\Theta^{1}(\eta)=\Theta_{\lambda}^{1}(\eta)$, we first find an interval $\left(\lambda_{l}, \lambda_{r}\right)$ containing $\lambda_{m l}$, and after that reveal the eigenvalue, e.g., by repeatedly bisecting the interval. At each step, functions $\Theta_{\lambda}^{0}(\eta)$ and $\Theta_{\lambda}^{1}(\eta)$ are computed as solutions of (28) subject 
to the initial conditions (30) and (31), respectively. Note that for a practical implementation, we have to truncate the series, the functions $\mathcal{A}(\eta)$ and $\mathcal{B}(\eta)$ are expressed by. In order to guarantee the desired accuracy, the estimates (11) and (16) can be utilized.

\subsubsection{Auxiliary functions}

After the eigenvalue $\lambda_{m l}$ has been found, the following algorithm allows to compute $T_{m l}(\eta)$ normalized 'in advance' according to (5) .

In addition to the Prüfer angle $\Theta_{\lambda_{m l}}^{0}(\eta)=\Theta_{\lambda_{m l} l}^{1}(\eta)=\Theta(\eta)$ we introduce an auxiliary function $\Upsilon(\eta)$, which plays the role of 'an amplitude'. At each point $\eta \in\left[\delta_{0}, 1-\delta_{1}\right]$ the eigenfunction $T_{m l}(\eta)$ along with its derivative can be expressed using $\Theta(\eta)$ and $\Upsilon(\eta)$ by the following relations:

$$
\begin{aligned}
T_{m l}(\eta) & =\Upsilon(\eta) \frac{\cos (\Theta(\eta))}{\nu(\eta)}, \\
\frac{\mathrm{d}}{\mathrm{d} \eta} T_{m l}(\eta) & =-\Upsilon(\eta) \frac{\nu(\eta) \sin (\Theta(\eta))}{\left(1-\eta^{2}\right)},
\end{aligned}
$$

respectively. Note that outside the interval $\left[\delta_{0}, 1-\delta_{1}\right]$, where the Prüfer angle varies too fast to be calculated accurately, the eigenfunction can be recovered from (30) and (31). Near $\eta=0$ we have

$$
\begin{aligned}
& T_{m l}(\eta)=T_{m l}(0) \eta^{m+1 / 2} \sum_{k=0}^{\infty} \tau_{k}^{0} \eta^{2 k}, \quad \eta \in\left[0, \delta_{0}\right], \\
& \tau_{0}^{0}=1, \quad \tau_{k}^{0}=\frac{1}{2 k}\left[\sum_{j=0}^{k-1} \tau_{j}^{0} \alpha_{k-j}+\left(m+2 k-\frac{3}{2}\right) \tau_{k-1}^{0}\right], k=1,2, \ldots
\end{aligned}
$$

Similarly,

$$
\begin{aligned}
& T_{m l}(\eta)=T_{m l}(1) \sum_{k=0}^{\infty} \tau_{k}^{1}(1-\eta)^{k}, \quad \eta \in\left[1-\delta_{1}, 1\right], \\
& \tau_{0}^{1}=1, \quad \tau_{k}^{1}=\frac{1}{2 k}\left[(k-1) \tau_{k-1}^{1}-\sum_{j=0}^{k-1} \tau_{j}^{1} \beta_{k-j}\right], \quad k=1,2, \ldots
\end{aligned}
$$


Back to the interval $\left[\delta_{0}, 1-\delta_{1}\right]$, one can easily show that $\Upsilon(\eta)$ satisfies the linear equation

$$
\frac{\mathrm{d} \Upsilon}{\mathrm{d} \eta}=\Upsilon V(\eta)
$$

where

$$
V(\eta)=\left[\frac{\mathrm{Q}(\eta)}{\nu^{2}(\eta)}-\frac{\nu^{2}(\eta)}{\left(1-\eta^{2}\right)}\right] \sin (\Theta) \cos (\Theta)+\frac{\nu^{\prime}(\eta)}{\nu(\eta)}\left[\cos ^{2}(\Theta)-\sin ^{2}(\Theta)\right]
$$

Moreover, we shall also need a function $\gamma(\eta)$, solving the adjoint equation,

$$
\frac{\mathrm{d} \gamma}{\mathrm{d} \eta}=-\gamma V(\eta)
$$

Evidently, for any solutions of $(37), \gamma(\eta) \Upsilon(\eta) \equiv$ const holds.

Among all solutions of (35), we are interested in the one which provides an eigenfunction normalized by (5). In order to find such solution, we introduce an additional auxiliary function $H\left(\eta, \eta_{i}\right)$ such that

$$
\int_{\eta_{i}}^{\eta} T_{m l}^{2}\left(\eta^{\prime}\right) \mathrm{d} \eta^{\prime}=H\left(\eta, \eta_{i}\right) \Upsilon(\eta), \quad \eta_{i} \in(0,1) .
$$

For a given arbitrary point $\eta_{\text {mid }} \in(0,1)$, let us assume that the values $H\left(\eta_{\text {mid }}, 0\right)$ and $H\left(\eta_{\text {mid }}, 1\right)$ are known. Then,

$$
\Upsilon\left(\eta_{\text {mid }}\right)=\left[H\left(\eta_{\text {mid }}, 0\right)-H\left(\eta_{\text {mid }}, 1\right)\right]^{-1 / 2},
$$

which follows immediately from the relation

$$
1=\int_{0}^{1} T_{m l}^{2}(\eta) \mathrm{d} \eta=\left[H\left(\eta_{m i d}, 0\right)-H\left(\eta_{m i d}, 1\right)\right] \Upsilon^{2}\left(\eta_{m i d}\right) .
$$

By direct verification, it follows

$$
\mathrm{d} H\left(\eta, \eta_{i}\right) / \mathrm{d} \eta=-2 H\left(\eta, \eta_{i}\right) V(\eta)+\frac{\cos ^{2}(\Theta)}{\nu^{2}(\eta)} .
$$

Also, the definition of $H$ yields at any point $\eta_{i} \in I$, in particular at $\eta_{i} \rightarrow+0$ and $\eta_{i} \rightarrow 1-0$,

$$
H\left(\eta_{i}, \eta_{i}\right)=0 .
$$

However, (40) cannot be integrated outside the interval $\left[\delta_{0}, 1-\delta_{1}\right]$, since the the Prüfer angle is not known there. Therefore, the 'natural' initial conditions 
are transferred from the singular points $\eta=0, \eta=1$ to $\eta=\delta_{0}$ and $\eta=1-\delta_{1}$ using (33) and (34), respectively. Truncated to $N_{i}, i=0,1$, the ultimate expressions for $H(\eta, 0)$ and $H(\eta, 1)$ become,

$$
\begin{aligned}
& H(\eta, 0)=\frac{\eta \cos ^{2}(\theta(\eta))}{\nu^{2}(\eta)}\left[\frac{\frac{1}{2(m+1)}+\sum_{k=0}^{N_{0}} \frac{\chi_{k}^{0}}{2(m+k+1)} \eta^{2 k}}{1+\sum_{k=0}^{N_{0}} \chi_{k}^{0} \eta^{2 k}}\right], \eta \in\left(0, \delta_{0}\right], \\
& H(\eta, 1)=-\frac{(1-\eta) \cos ^{2}(\theta(\eta))}{\nu^{2}(\eta)}\left[\frac{1+\sum_{k=0}^{N_{1}} \frac{\chi_{k}^{1}}{k+1}(1-\eta)^{k}}{1+\sum_{k=0}^{N_{1}} \chi_{k}^{1}(1-\eta)^{k}}\right], \eta \in\left[1-\delta_{1}, 1\right)
\end{aligned}
$$

where

$$
\chi_{k}^{i}=2 \tau_{k}^{i}+\left(\sum_{j=1}^{k-1} \tau_{j}^{i} \tau_{k-j}^{i}\right), \quad \zeta_{k}^{i}=\left(2 \tau_{k}^{i}+\sum_{j=1}^{k-1} \tau_{j}^{i} \tau_{k-j}^{i}\right) .
$$

Summarizing the above consideration, the evaluation of the eigenfunction $T_{m l}(\eta)$ at a grid $\Delta_{h}$ is carried out as follows. On each subinterval $I_{j}=$ $\left(\eta_{j}, \eta_{j+1}\right), j=0,1, \ldots,[n / 2]-1$, we integrate the system of equations (28), (37), and (40) with the initial conditions following for the functions $\Theta(\eta)$ and $H(\eta, 0)$ from the conditions (30) and $(41)$, while for the solution of (37), we require $\gamma_{j}\left(\eta_{j}\right)=1$. Finally, the values $\Theta\left(\eta_{j+1}\right), H\left(\eta_{j+1}, 0\right)$, and $\gamma_{j}\left(\eta_{j+1}\right)$ are stored.

Then for $j=n, n-1, \ldots,[n / 2]+1$, the same equations are integrated from $\eta_{j}$ to $\eta_{j-1}$, again, each time with the natural initial conditions following from the relations (31) and (41) for the functions $\Theta(\eta)$ and $H(\eta, 1)$, respectively, and with $\gamma_{j}\left(\eta_{j}\right)=1$. This time we store the values $\Theta\left(\eta_{j-1}\right), H\left(\eta_{j-1}, 1\right)$ and $\gamma_{j}\left(\eta_{j-1}\right)$.

Now (39) yields the desired value for the amplitude at the center of the computational interval, $\Upsilon\left(\eta_{[n / 2]}\right)$, so that at any other point $\eta_{j}$ the amplitude is

$$
\begin{array}{ll}
\Upsilon\left(\eta_{j}\right)=\Upsilon\left(\eta_{j+1}\right) \gamma_{j}\left(\eta_{j+1}\right) & j=[n / 2]-1,[n / 2]-2, \ldots, 0, \\
\Upsilon\left(\eta_{j}\right)=\Upsilon\left(\eta_{j-1}\right) \gamma_{j}\left(\eta_{j-1}\right) & j=[n / 2]+1,[n / 2]+2, \ldots, n,
\end{array}
$$


which follows from (35), (37) and the above initial conditions for $\gamma_{j}(\eta)$. Substituting the values $\Theta\left(\eta_{j}\right)$ and $\Upsilon\left(\eta_{j}\right)$ into (32), we obtain the normalized eigenfunction $T_{m l}(\eta)$ and its derivative computed to the accuracy defined by the numerical integration procedure.

In our calculations we usually employ the standard fourth order Runge-Kutta method with adaptive stepsize control. The attractive feature of the proposed approach is that the accuracy requirements, as well as the possible complicated behavior of the solution (e.i., highly oscillating eigenfunction) does not influence the amount of the stored data, the relation between the multi-index $(m, l)$ and the calculated pair of eigenvalue and eigenfunction is transparent, no unnecessary eigenvalues or eigenfunctions are calculated. An advantage is also that the above procedure can without any change be performed on a non-equidistant grid. This approach is especially useful, in case of highly oscillating eigenfunctions. However, the application area is restricted to the cases where eigenfunction oscillations are not localized far away from the ends of the interval $I$. The cases discussed in Section 4, cf. Figures 4 to 6 , cause an essential difficulty for the above technique. In the next section, we compare the results obtained via Approaches 1 and 2 with those provided by the Approach 3, cf. [29]. Moreover, we discuss the convergence properties of the finite difference schemes when they are applied to solve problems with singularities. As already mentioned, we expect to see order reductions in case when the higher derivatives of the analytical solution are unsmooth.

\section{Numerical experiments}

For the numerical experiments two MATLAB codes realizing the finite difference schemes of order 4 and 6 have been implemented. All runs have been carried out on grids $\Delta_{h}$ with a constant stepsize $h$. It is quite clear that a variable stepsize will greatly improve the performance of the method but this question was not in focus of the present investigation. Here, we were rather interested to find out in which cases Approaches 1 and 2 can be viewed as a good alternative to Approach 3. For the first approach $\left(\eta_{0}=0\right)$ we use the MATLAB function eigs to solve the related algebraic eigenvalue problem providing in a very efficient way the first eigenvalues and eigenvectors. In the second approach, since the left boundary condition in $\eta_{0}>0$ depends nonlinearly on the eigenvalue, the discrete system is nonlinear and therefore, the eigenvalue and the associated eigenfunction are computed by means of the Newton iteration. The first approach is at the moment considerably faster than the second one (for the same values of $n$ ). 
We first calculate the solution of the eigenvalue problem for various choices of the parameters $c, m$ and $l$. We use the first approach when $m>0$ and the second approach with $\eta_{0}=4 \mathrm{e}-3$ when $m=0$. The results of these simulations are compared with those found in [29].

Table 1: Numerical values for $\Lambda=\lambda+c^{2}$ obtained with a method of order 6 .

\begin{tabular}{|r|c|r|c|r|c|}
\hline$c$ & $\Lambda_{0,0}(n=10000)$ & $c$ & $\Lambda_{1,2}(n=5000)$ & $c$ & $\Lambda_{2,3}(n=3500)$ \\
\hline 0.1 & $7.549989717 \mathrm{e}-1$ & 1 & $3.626510128 \mathrm{e}+1$ & 1 & $8.127528992 \mathrm{e}+1$ \\
1 & $1.239593273 \mathrm{e}+0$ & 2 & $3.782030980 \mathrm{e}+1$ & 2 & $8.285466755 \mathrm{e}+1$ \\
2 & $2.585796843 \mathrm{e}+0$ & 5 & $4.916003732 \mathrm{e}+1$ & 5 & $9.406500738 \mathrm{e}+1$ \\
5 & $8.586917680 \mathrm{e}+0$ & 10 & $9.595581537 \mathrm{e}+1$ & 10 & $1.367942028 \mathrm{e}+2$ \\
10 & $1.869011000 \mathrm{e}+0$ & 14 & $1.465750584 \mathrm{e}+2$ & 18 & $2.763539366 \mathrm{e}+2$ \\
\hline
\end{tabular}

In Table 1 we recalculate the eigenvalues specified in [29, Table I]. For all considered parameter values, the results shown in Table 1 are in a perfect agreement with those in [29, Table I]. Some of the numbers differ only by one unit of the last digit, which means that their relative error is smaller than $1 \mathrm{e}-8$. The most work intensive, with respect to the required number of mesh points, is the case $m=0$.

Table 2: Numerical approximation for $T_{0,0}\left(\eta_{i}\right)$ obtained by the Approach 2 with $n=15000$ and $\eta_{0}=1 e-3$.

\begin{tabular}{|c|c|c|c|c|}
\hline$\eta_{i}$ & $c=1$ & $c=2$ & $c=5$ & $c=10$ \\
\hline 0.1 & $4.7463759 \mathrm{e}-1$ & $5.5542096 \mathrm{e}-1$ & $9.1566287 \mathrm{e}-1$ & $1.3145513 \mathrm{e}+0$ \\
0.2 & $6.6877647 \mathrm{e}-1$ & $7.7470577 \mathrm{e}-1$ & $1.2203224 \mathrm{e}+0$ & $1.6224792 \mathrm{e}+0$ \\
0.3 & $8.1407035 \mathrm{e}-1$ & $9.2709557 \mathrm{e}-1$ & $1.3516563 \mathrm{e}+0$ & $1.5768955 \mathrm{e}+0$ \\
0.4 & $9.3194797 \mathrm{e}-1$ & $1.0360733 \mathrm{e}+0$ & $1.3510305 \mathrm{e}+0$ & $1.3042911 \mathrm{e}+0$ \\
0.5 & $1.0304405 \mathrm{e}+0$ & $1.1101146 \mathrm{e}+0$ & $1.2462605 \mathrm{e}+0$ & $9.3164207 \mathrm{e}-1$ \\
0.6 & $1.1135091 \mathrm{e}+0$ & $1.1535345 \mathrm{e}+0$ & $1.0666060 \mathrm{e}+0$ & $5.7032801 \mathrm{e}-1$ \\
0.7 & $1.1834131 \mathrm{e}+0$ & $1.1692138 \mathrm{e}+0$ & $8.4347613 \mathrm{e}-1$ & $2.9133209 \mathrm{e}-1$ \\
0.8 & $1.2415737 \mathrm{e}+0$ & $1.1595679 \mathrm{e}+0$ & $6.0784638 \mathrm{e}-1$ & $1.1707804 \mathrm{e}-1$ \\
0.9 & $1.2889569 \mathrm{e}+0$ & $1.1269505 \mathrm{e}+0$ & $3.8697123 \mathrm{e}-1$ & $3.1974276 \mathrm{e}-2$ \\
1.0 & $1.3262673 \mathrm{e}+0$ & $1.0738288 \mathrm{e}+0$ & $2.0153293 \mathrm{e}-1$ & $3.0016112 \mathrm{e}-3$ \\
\hline
\end{tabular}

In Tables 2 to 4 , we report on the values of the eigenfunctions $T_{m l}$ (at points $\left.\eta_{i}=0.1, \ldots, 1\right)$ corresponding to the eigenvalues displayed in Table 1 . 
Table 3: Numerical approximation for $T_{1,2}\left(\eta_{i}\right)$ obtained by the Approach 1 with $n=7500$.

\begin{tabular}{|c|r|r|r|r|}
\hline$\eta_{i}$ & $c=1$ & $c=2$ & $c=5$ & $c=10$ \\
\hline 0.1 & $3.1757219 \mathrm{e}-1$ & $3.2337538 \mathrm{e}-1$ & $3.5845228 \mathrm{e}-1$ & $4.6132749 \mathrm{e}-1$ \\
0.2 & $7.8903773 \mathrm{e}-1$ & $7.9845587 \mathrm{e}-1$ & $8.4503352 \mathrm{e}-1$ & $8.8440506 \mathrm{e}-1$ \\
0.3 & $1.1392876 \mathrm{e}+0$ & $1.1392393 \mathrm{e}+0$ & $1.1002673 \mathrm{e}+0$ & $6.9329104 \mathrm{e}-1$ \\
0.4 & $1.1626804 \mathrm{e}+0$ & $1.1369972 \mathrm{e}+0$ & $9.0983080 \mathrm{e}-1$ & $-8.1338551 \mathrm{e}-2$ \\
0.5 & $7.4636717 \mathrm{e}-1$ & $6.8819422 \mathrm{e}-1$ & $2.6040225 \mathrm{e}-1$ & $-8.6019220 \mathrm{e}-1$ \\
0.6 & $-6.4193513 \mathrm{e}-2$ & $-1.4015609 \mathrm{e}-1$ & $-6.1834886 \mathrm{e}-1$ & $-9.7298098 \mathrm{e}-1$ \\
0.7 & $-9.9083021 \mathrm{e}-1$ & $-1.0450076 \mathrm{e}+0$ & $-1.2795870 \mathrm{e}+0$ & $-2.0100170 \mathrm{e}-1$ \\
0.8 & $-1.4441327 \mathrm{e}+0$ & $-1.4298020 \mathrm{e}+0$ & $-1.1558726 \mathrm{e}+0$ & $1.0243617 \mathrm{e}+0$ \\
0.9 & $-4.4333317 \mathrm{e}-1$ & $-3.6047550 \mathrm{e}-1$ & $2.9275304 \mathrm{e}-1$ & $1.9247110 \mathrm{e}+0$ \\
1.0 & $3.4633569 \mathrm{e}+0$ & $3.4606674 \mathrm{e}+0$ & $3.4142845 \mathrm{e}+0$ & $1.9405949 \mathrm{e}+0$ \\
\hline
\end{tabular}

Again these results perfectly agree with those given in [29, Table II]. The required number of meshpoints to solve the problem to a prescribed accuracy decreases with increasing $m$. Due to unsmooth higher solution derivatives and consequently, due to order reductions, Approach 1 is not suitable for $m=0$ because it becomes extremely inefficient. For Approach 2, 10000 points were necessary to obtain the sufficiently accurate solution.

In Figures 1 to 3, we illustrate the convergence behavior for Approaches 1 and 2 and $m=0,1$ and 2 . We use methods of order 4 and 6 to solve problem (3) with $c=100$ and the previous combinations of $m$ and $l$. The second approach performs better than the first one when $m=0,1$ while the performance becomes comparable for $m \geq 2$. The reason for this behavior can be explained as follows: In Approach 1 the differential equation (3) is treated on the whole interval $[0,1]$ including the singular point $\eta_{0}=0$. This means that for small values of $m$ higher solution derivatives become unbounded when $\eta$ approaches zero. This results in an order reduction down to $p=2 m$ for $m>0$, while for $m=0$ the convergence is sublinear. Approach 2 avoids the singular point, the higher derivatives stay bounded on $[\eta, 1]$ (although very large) and therefore, the convergence is more regular. The right singular point $\eta=1$ does not effect the convergence of the scheme. 

(a) Approach 1 .
(b) Approach 2 .

Figure 1: Convergence behavior of the difference schemes of consistency order 4 (solid blue line) and order 6 (solid green line) applied to solve problem (3) with $c=100$ and $m=l=0$. Dotted lines illustrate the behavior $O\left(h^{r}\right)=O\left(\left(\frac{1}{n}\right)^{r}\right)$ for different values of $r$. When using Approach 1, both methods suffer from order reduction, to an order lying between $\frac{1}{9}$ and $\frac{1}{8}$. Indeed the global error of both methods is $O\left(h^{0.118}\right)$, see left graph. The right graph also suggests slight order reductions; the asymptotic behavior of the global error of the 4th and 5th order scheme is $O\left(h^{3.6}\right)$ and $O\left(h^{5.4}\right)$, respectively. 

(a) Approach 1.
(b) Approach 2 .

Figure 2: Convergence behavior of the difference schemes of consistency order 4 (solid blue line) and order 6 (solid green line) applied to solve problem (3) with $c=100, m=1$ and $l=2$. Dotted lines illustrate the behavior $O\left(h^{r}\right)=O\left(\left(\frac{1}{n}\right)^{r}\right)$ for different values of $r$. When using Approach 1, both methods suffer from order reduction; their global errors are $O\left(h^{2}\right)$, see left graph. The right graph also shows order reductions; The global errors are $O\left(h^{3.4}\right)$ and $O\left(h^{4.8}\right)$ for the methods of order 4 and 6 , respectively. 

(a) Approach 1.
(b) Approach 2 .

Figure 3: Convergence behavior of the difference schemes of consistency order 4 (solid blue line) and order 6 (solid green line) applied to solve problem (3) with $c=100, m=2$ and $l=3$. Dotted lines illustrate the behavior $O\left(h^{r}\right)=O\left(\left(\frac{1}{n}\right)^{r}\right)$ for different values of $r$. When using Approach 1, only the 6 th order method suffers from order reduction down to 4, see left graph. In the right graph, again order reduction for the 6th order scheme can be observed; the respective global error is $O\left(h^{4.4}\right)$.

Finally, we numerically simulated (3) for the case where the eigenfunctions show an oscillatory behavior near the left end or the right end of the integration interval, $[0,1]$, and vanish elsewhere, cf. Figures 4 to 6 . For this type of difficulty, analytical techniques proposed in Approach 3, have problems in providing the approximation of the solution of (3). For this simulation, schemes of order 6 has been used and the number of points was varying from 1000 to 10000. Since in this case no data in [29] is available, we have checked the accuracy of the approximation by calculating the values of the integral

$$
I_{m l}(\eta)=\int_{0}^{1} \sqrt{c \eta \rho} J_{m}(c \eta \rho) T_{m l}(\rho) \mathrm{d} \rho,
$$

cf. (2), which is approximated by the composite Simpson's rule in order to compare its value with $\mu_{m l} T_{m l}(\eta)$. Note that the eigenvalues $\mu_{m l}$ remain unknown before we compute the ratio $I_{m l}(\eta) / T_{m l}(\eta)$ and check if it remains constant on the interval $(0,1)$, which then confirms that $T_{m l}(\eta)$ is a FHT eigenfunction. 
Moreover, we also provide the values of the maximal deviation, $\| \mu_{m l} T_{m l}-$ $I_{m l} \|_{\infty}$. Since the values of $\mu_{m l} T_{m l}$ and $I_{m l}$ are given only in the $n+1$ grid points, the above norm is the discrete maximum norm in $\mathbb{R}^{n+1}$.

Figures 4 to 6 show the results for the method of the (consistency) order 6 . One can see, cf. subgraphs (b), (c), and (d), that the convergence of the method and the solution accuracy are quite satisfactory.

As mentioned above, the results of calculations using Approaches 1 and 2 were compared with those performed via Approach 3 coded in C. Note that in the first two approaches relative accuracy, while in Approach 3 the absolute accuracy is controlled.

The aim of the numerical tests was to verify the validity of all presented numerical schemes and to determine the application area of each of them.

The simulations showed that all approaches under consideration worked perfectly fine in a wide range of parameters $c, m, l \leq 100$. With growing eigenfunction index, calculations carried out using the first two approaches require finer meshes and become more time consuming than Approach 3. The situation is redoubled by the fact that the eigenvalues $\lambda_{m l}$ increase with $l \rightarrow \infty$, so that one needs higher relative accuracy in order to guarantee the same absolute accuracy.

On the other hand, Approach 3 works very well in cases when the key parameter values are comparable. In case $c \gg 1$, say $c=100$ and small $l$ it is not applicable, since the numerical integration of (29) becomes unstable.

Another drawback of the Approach 3 is that it is very sensitive with respect to the choice of the points $\eta=\delta_{0}$ and $\eta=1-\delta_{1}$. If they are too close to the singular ends of the interval $(0,1)$, the right-hand sides of (29) and (37) become too large to provide high absolute accuracy with a reasonable step size. Approaches 1 and 2 are free from those disadvantages. Note that for small indexes $l$ one does not need high relative accuracy in order to achieve high absolute accuracy. 

(c) Error with $n=5000$.
(d) Error with $n=10000$.

Figure 4: Method of order 6: Numerical solution and its accuracy provided by Approach 2 for $m=0, l=3$ and $c=100$. 

(c) Error with $n=5000$.
(d) Error with $n=10000$.

Figure 5: Method of order 6: Numerical solution and its accuracy provided by Approach 2 for $m=0, l=3$ and $c=200$. 

(c) Error with $n=5000$.
(d) Error with $n=10000$.

Figure 6: Method of order 6: Numerical solution and its accuracy provided by Approach 1 for $m=50, l=3$ and $c=50$.

\section{Conclusions}

We have presented three different approaches for the approximation of the eigenfunctions and eigenvalues of the Finite Hankel Transform. In order to avoid the computational difficulties caused by a specific behaviour of FHT eigenvalues, a self-adjoint Sturm-Liouville problem (3), (4), is solved 
numerically, which produces the same set of eigenfunctions whose associated eigenvalues are well resolved. The Sturm-Liouville problem related to FHT is singular, the definition domain of the Sturm-Liouville operator is described from the requirement on the analytical solution to stay bounded on $[0,1]$.

Boundary condition transfer from the singular point to a close regular point allows to formulate a regular Sturm-Liouville problem which inside the truncated interval is equivalent to the original singular one.

By a limiting procedure the equivalent boundary conditions are then transferred back to singular points and the Sturm-Liouville problem is reformulated in the form suitable for numerical implementation.

For numerical solution of the the problem posed on the whole interval $[0,1]$ Approach 1 is proposed, while Approaches 2 and 3 deal with the truncated regular Sturm-Liouville problems, that avoid order reductions of the numerical method caused by insufficient smoothness of the eigenfunctions at $\eta=0$. Yet, Approach 1 is evidently simpler and does not require extra calculations outside the truncated interval. Both Approaches 1 and 2 use high order finite difference schemes and are convenient for numerical implementation with MATLAB and easy for accuracy analysis. At present, both approaches are implemented on an equidistant grid, whose step size depends dramatically on the index $l$ responsible for the oscillations in the eigenfunctions. Unlike the first two approaches, the third one based on the Prüfer angle refines the computational grid automatically and adaptively. It is coded in $\mathrm{C}++$ and does not depend on other computational software.

All presented approaches are efficient, accurate and robust; they are applicable in a wide range of parameters $c, m, l$. We emphasize that they all can be used with values of the key parameter $c$ relevant in practice.

Each of the presented approaches has a specific area of application where others do not work or can not provide the required accuracy efficiently. For $m=0$ Approach 2 yields better accuracy than Approach 1. For the evaluation of eigenfunctions of small index $l$ and $c \gg 1$, we recommend to use Approaches 1 and 2, while for highly oscillating eigenfunctions Approach 3 is more advantageous than the other ones.

The authors are very grateful for the warm hospitality of Vienna University of Technology while working on this paper. 


\section{References}

[1] A.A. Abramov, A.L. Dyshko, N.B. Konyukhova, and T.V. Levitina, Computation of radial wave functions for spheroids and triaxial ellipsoids by the modified phase function method, Comput. Math. Math. Phys. 31, pp. 25-42 (1991).

[2] A.A. Abramov, A.L. Dyshko, N.B. Konyukhova, T.V. Pak, and B.S. Pariiskii, Evaluation of prolate spheroidal function by solving the corresponding differential equations, U.S.S.R. Comput. Math. Math. Phys. 24, pp. 1-11 (1984).

[3] C. Aime, Radon approach to shaped and apodized apertures for imaging exoplanets, Astron. \& Astrophys. 434, pp. 785-794 (2005).

[4] C. Aime, Apodized apertures for solar coronagraphy, Astronom. \& Astrophys. 467, pp.317-325 (2007).

[5] P. Amodio and G. Settanni, High Order Finite Difference Schemes for the Numerical Solution of Eigenvalue Problems for IVPs in ODEs, Numerical Analysis and Applied Mathematics, AIP Conference Proceedings 1281, pp. 202-205 (2010).

[6] P. Amodio and G. Settanni, A matrix method for the solution of SturmLiouville problems JNAIAM J. Numer. Anal. Indust. Appl. Math., in press.

[7] P. Amodio and I. Sgura, High-order finite difference schemes for the solution of second-order BVPs, J. Comput. Appl. Math. 176, pp. 59-76 (2005).

[8] W. Auzinger, E. Karner, O. Koch, and E.B. Weinmüller, Collocation methods for the solution of eigenvalue problems for singular ordinary differential equations, Opuscula Math. 26, pp. 229-241 (2006).

[9] V.N. Beskrovny and M.I. Kolobov, Quantum-statistical analysis of superresolution for optical systems with circular symmetry, Phys. Rev. A 78, 043824 (2008).

[10] R.F. Boivin, Eigenfunctions of the Fourier Transformation over a Circle, Part 1: Approximation of Sturmian Eigenvalues, DRDC Ottawa Technical Memorandum 342, 2008. 
[11] G. Borgiotti, Hyperspheroidal functions-high beam efficiency illumination for circular antennas, Antennas and Propagation Society International Symposium, pp. 30-39 (1969).

[12] G.R. Boyer, Pupil filters for moderate superresolution, Appl. Optics 15, pp. 3089-3093 (1976).

[13] L. Brugnano and D. Trigiante, Solving Differential Problems by Multistep Initial and Boundary Value Methods, Gordon and Breach Science Publishers, Amsterdam, 1998.

[14] E.A. Coddington, N. Levinson, Theory of differential equations, New York, McGraw-Hill, 1955.

[15] R. Hammerling, O. Koch, C. Simon, E.B. Weinmüller, Numerical solution of singular ODE eigenvalue problems in electronic structure computations, J. Comput. Phys. 181, pp. 1557-1561 (2010).

[16] J.C. Heurtley, Hyperspheroidal Functions-Optical Resonators with Circular Mirrors, Quasi-Optics, Proc. Symposium on Quasi-Optics, June 8-10, 1964, New York, Vol. 14 of the Microwave Research Institute Symposia Series, Polytechnic Press, Polytechnic Institute of Brooklyn, Brooklyn, NY, p. 367 (1964).

[17] F.R. de Hoog and R. Weiss, Difference methods for boundary value problems with a singularity of the first kind, SIAM J. Numer. Anal. 13, pp. 775-813 (1976).

[18] A. Karoui, Unidimensional and bidimensional prolate spheroidal wave functions and applications, J. Franklin Inst. 348, pp. 1668-1694 (2011).

[19] A. Karoui and T. Moumni, Spectral analysis of the finite Hankel transform and circular prolate spheroidal wave functions, J. Comput. Appl. Math. 233, pp. 315-333 (2009).

[20] A. Klug and R.A. Crowther, Three-dimensional Image Reconstruction from the Viewpoint of Information Theory, Nature 238, pp. 435-440 (1972).

[21] I.V. Komarov, L.I. Ponomarev, and S.Y. Slavyanov, Spheroidal and Coulomb Spheroidal Functions, (in Russian), Nauka, Moscow, 1976.

[22] N.V. Kuznetsov, On eigen-functions of an integral equation, Mathematical problems in the theory of wave propagation, Part 3, Zap. Nauchn. Sem. LOMI, Nauka, Leningrad, pp. 66-150 (1970). 
[23] B. Larsson, T.V. Levitina, and E.J. Brändas, On Generalized Prolate Spheroidal Functions, Proc. CMMSE-2002, Alicante, Spain II, pp. 220223 (2002).

[24] W.P. Latham and M.L. Tilton, Calculation of prolate functions for optical analysis, Appl. Optics 26, pp. 2653-2658 (1987).

[25] T.V. Levitina and E.J. Brändas, Computational Techniques for Prolate Spheroidal Wave Functions in Signal Processing, J. Comp. Meth. Sci. Eng. 1, pp. 287-313 (2001).

[26] A.K. Louis, Nonuniqueness in inverse Radon problems: the frequency distribution of the ghosts, Math. Z. 185, pp. 429-440 (1984).

[27] D. Rhodes, On the aperture and pattern space factors for rectangular and circular apertures, IEEE Trans. Antennas and Propagation 19, pp. 763770 (1971).

[28] S.S. Sherif, M.R. Foreman, and P. Török , Eigenfunction expansion of the electric fields in the focal region of a high numerical aperture focusing system, Optics Express 16, pp. 3397-3407 (2008).

[29] D. Slepian, Prolate spheroidal wave functions, Fourier analysis and uncertainty, IV: Extensions to many dimensions; generalized prolate spheroidal functions, Bell System Tech. J. 43, pp. 3009-3058 (1964).

[30] D. Slepian and H.O. Pollak, Prolate spheroidal wave functions, Fourier analysis and uncertainty I, Bell System Tech. J. 40, pp. 43-64 (1961).

[31] K.C. Tam, V. Perez-Mendez, and B. MacDonald, Limited angle 3-D reconstructions from continuous and pinhole projections, IEEE Trans. Nucl. Sci. 27 pp. $445-458$ (1980).

[32] L.A. Weinstein, Open Resonators and Open Waveguides, Golem, Boulder, 1969.

[33] X. Zhang, Wavenumber Spectrum of Very Short Wind Waves: An Application of Two-Dimensional Slepian Windows to Spectral Estimation, J. Atmos. Oceanic Technol. 11, pp. 489-505 (1994). 